\title{
Will the carboplatin-paclitaxel-bevacizumab combination become the preferred option as the first line in advanced cervical cancer? A small experience from a single oncologic Center
}

\author{
Raquel Bratos Lorenzo ${ }^{1}$, Luisa Sánchez Lorenzo ${ }^{1}$, Antonio González-Martín ${ }^{1}$
}

\begin{abstract}
Background Despite the widespread introduction of screening programs, cervical cancer remains an important health problem, particularly in developing countries. Treatment options for patients with metastatic cancer and persistent or recurrent disease after curative therapy are limited and represent an unmet medical need.

Patients and Methods We report three clinical cases of metastatic cervical cancer in women treated with the combination of carboplatin, paclitaxel and bevacizumab. We also discuss current and future therapy for metastatic or recurrent cervical cancer, including ongoing investigations into the addition of bevacizumab to carboplatin/paclitaxel chemotherapy and the role of bevacizumab as maintenance therapy.

Results and Conclusion All three women received clinical benefit from the combination, including one complete and two partial responses. The tolerability of bevacizumab was excellent. Carboplatin/paclitaxel appears to be an acceptable and better tolerated alternative to cisplatin/paclitaxel in advanced or recurrent/persistent disease, and the carboplatin/paclitaxel/bevacizumab combination is under investigation for metastatic, recurrent or persistent cervical cancer in clinical trials such as the CECILIA trial (NCT02467907); the awaited results will add to the evidence for the use of this combination chemotherapy regimen in advanced cervical cancer.
\end{abstract}

Key words: metastatic cervical cancer, angiogenesis, bevacizumab, carboplatin, paclitaxel

\section{Case Reports}

\section{Case 1}

A 54-year-old female was diagnosed at age 47 with cervical adenocarcinoma International Federation of Gynecology and Obstetrics (FIGO) stage IB2. A retroperitoneal lymphadenectomy was carried out and 12 of 16 nodes were positive. She received weekly cisplatin-based chemoradiation therapy between June and July 2009 followed by vaginal brachytherapy. Thereafter, she received four cycles of adjuvant cisplatin-gemcitabine, finishing in September 2009. In July 2012, a nodal relapse in the retrocrural region and in her left supraclavicular area was detected. The multidisciplinary team agreed to remove the abdominal lymph node and to administer intensity modulated radiation therapy (IMRT) over the supraclavicular disease combined with four cycles of carboplatin-paclitaxel, which finished in January 2013.

Follow-up was normal until January 2015, when a new pathological mediastinal node was detected and afterwards removed by thoracic surgery.

In September 2015, multiple nodal relapse was detected and it was decided to start treatment with carboplatin AUC 5, paclitaxel $175 \mathrm{mg} / \mathrm{m}^{2}$ and bevacizumab $15 \mathrm{mg} / \mathrm{kg}$, which are the standard doses at our institution for this combination. A complete response was demonstrated after six cycles, and she continued 3-weekly bevacizumab as maintenance. Clinical tolerance was excellent, with the exception of grade 2 hypertension. The patient is still in complete response and receiving bevacizumab as maintenance therapy.

\section{Case 2}

The second patient is a 75-year-old woman diagnosed with a cervical adenocarcinoma involving the rectum, with lim-

${ }^{1}$ Medical Oncology Department and Gynecologic Oncology Unit, MD Anderson Cancer Center Hospital, Madrid, Spain.

Correspondence to:

Antonio González Martin,

MD Anderson Cancer Center,

C/ Arturo Soria 270, 28033 Madrid, Spain.

Phone: +34 917878605 - Fax: +34 917680687

E-mail: agonzalezm@seom.org

CANCER BREAKING NEWS 2017;5(1):21-24

DOI: $10.19156 / \mathrm{cbn} .2017 .0035$ 
ited pelvic peritoneal carcinomatosis and pelvic/paraaortic nodal infiltration (FIGO stage IVA at diagnosis). She was a screening failure for the CECILIA trial, due to rectum involvement demonstrated in magnetic resonance imaging (MRI). In December 2015 she started on 3-weekly carboplatin-paclitaxel. Computed tomography and MRI after three cycles showed a partial response. Thereafter, an endoscopic study showed a free rectum, and bevacizumab was added. Three additional cycles were administered, followed by 3-weekly maintenance bevacizumab until December 2016, when bone and lung metastatic progression were detected (i.e., a progression-free interval of 9 months). At that time, a phase I clinical trial with a new anti-PD-L1 therapy was offered, and she was included in the trial. Patient tolerability to bevacizumab was excellent.

\section{Case 3}

The third patient was diagnosed in 2010 at the age of 48 years with stage IB1 squamous cervical cancer. She was treated with radical hysterectomy and pelvic lymphadenectomy. Two years later, a vaginal recurrence was identified and radical chemo-radiation therapy was administered up until October 2012. Thirteen months later, a new pelvic relapse was diagnosed, and radical surgery with a pelvic exenteration and "neobladder reconstruction" was carried out. Pathological examination revealed G2 squamous carcinoma in the vaginal wall, bladder wall, pelvic peritoneum and small bowel (ileum). After discussion within the tumor board and with the patient, systemic therapy with six cycles of cisplatin $50 \mathrm{mg} / \mathrm{m}^{2}$ and paclitaxel $175 \mathrm{mg} / \mathrm{m}^{2}$ was proposed, which she received from March 2014 to July 2014.

In March 2016, peritoneal carcinomatosis with pelvic and retroperitoneal nodal involvement were detected. She started systemic treatment with the regimen of carboplatin, paclitaxel and bevacizumab. After six cycles she obtained a partial response and maintenance therapy with bevacizumab was continued until December 2016, when disease progression was diagnosed, with peritoneal carcinomatosis and new liver metastasis (i.e., a progression-free survival [PFS] of 9 months). The patient reported no significant adverse events. Subsequently, she was included in the phase I trial with the anti-PD-L1 therapy already mentioned.

\section{Current therapy for metastatic or recurrent cervical carcinoma}

Despite of the introduction of universal screening with cytology (the Papanicolau [Pap] test), DNA testing for highrisk human papillomavirus (HPV) types, and vaccination programs, cervical cancer remains an important health problem, mainly in developing countries [1].
Early stage and locally advanced cervical cancer may potentially be cured by surgery, radiation therapy and/ or chemoradiation with platinum-based chemotherapy. However, the prognosis for metastatic cancer and persistent or recurrent disease after chemoradiation remains poor, the limited options for these patients represent an unmet medical need.

\section{Beyond cisplatin in monotherapy}

Cisplatin $50 \mathrm{mg} / \mathrm{m}^{2}$ every 3 weeks was for two decades the standard of care for palliative systemic therapy in those patients that were candidate for chemotherapy. Nevertheless, the global efficacy was disappointing due to a low response rate (RR) (around 20\%), short median PFS (2.8-3.2 months) and overall survival (OS) (6.2-8.0 months).

Two randomized trials, the Gynecologic Oncology Group (GOG) 169 and GOG 179 trials, evaluated the efficacy and safety profile of cisplatin-based doublets (cisplatin plus paclitaxel or cisplatin plus topotecan) in comparison to cisplatin in monotherapy $[2,3]$. Both cisplatin-based combinations showed a superior RR and PFS. However, only the combination of cisplatin and topotecan improved OS. In a cross comparison of both trials, patients who had previously received radio-sensitizing platinum-based chemotherapy, achieved a higher RR with the combination of cisplatin plus paclitaxel compared to the cisplatin and topotecan doublet. Based on this finding, the combination of paclitaxel and cisplatin was considered the reference regimen for future clinical trials in metastatic cervical cancer by the GOG.

Subsequently, GOG 204 was a phase III trial designed to evaluate the optimal cisplatin doublet among the population with metastatic cervical cancer [4]. Four platinumbased intravenous regimens were studied, including paclitaxel-cisplatin (PC) (as control arm), vinorelbine-cisplatin (VC), gemcitabine-cisplatin (GC) and topotecan-cisplatin (TC). None of the experimental combinations was superior in terms of RR, OS and PFS, compared with the control arm (PC). However, the results observed for OS in the PC arm (12.9 months) compared with the other three arms (10-10.3 months), shaped the support for the cisplatin-paclitaxel combination that also became the preferred regimen in clinical practice for metastatic or recurrent cervical carcinoma.

Although the combination of cisplatin-paclitaxel has been considered the standard of care, the carboplatin-paclitaxel regimen may offer a convenient alternative based on a more favorable toxicity profile consisting of less nausea/ vomiting, nephropathy and a lower rate of neuropathy. This better safety profile results in a much more attractive regimen, from our clinical point of view. However, the 
evidence for the use of carboplatin instead of cisplatin is based on only one clinical trial conducted in Japan. The Japan Gynecologic Oncology Group (JGOG) carried out a randomized phase III trial (JGOG 0505) to confirm that the carboplatin/paclitaxel combination was not inferior to cisplatin/paclitaxel in terms of OS in patients who had one or less previous platinum containing regimen and no prior taxane. The study reached its primary endpoint demonstrating non-inferiority in OS between both regimens [5]. However, OS was shorter in the carboplatin-paclitaxel arm (13 months) than in the cisplatin-paclitaxel group (23.2 months) (hazard ratio [HR] 1.571, 95\% confidence interval [CI] 1.06-2.32) in patients who had not received prior cisplatin-based chemotherapy. On the other hand, carboplatin-paclitaxel was more effective than cisplatinpaclitaxel for patients who had been treated with a previous platinum-based regimen.

Based on this trial, the paclitaxel-carboplatin regimen could be considered as an alternative option for advanced or recurrent/persistent cervical cancer, especially in patients that have previously received cisplatin.

\section{Angiogenesis in cervical cancer}

Angiogenesis is crucial for the initiation, proliferation and progression of cervical cancer, making this process a potential target for therapy [6]. Bevacizumab is a fully humanized monoclonal antibody blocking the vascular endothelial growth factor (VEGF) that had demonstrated activity in patients with recurrent disease previously treated. A retrospective analysis of heavily pretreated patients with recurrent cervical cancer showed a clinical benefit rate of $67 \%$ and a favorable toxicity profile when bevacizumab was combined with chemotherapy [7]. In addition, the phase II GOG-227C trial evaluated bevacizumab in patients with recurrent or persistent squamous cell cervical carcinoma and one or two prior cytotoxic combinations. The medians of PFS and OS were 3.4 months $(95 \%$ CI 2.53-4.53) and 7.3 months (95\% CI 6.11-10.41), respectively [6].

The role of bevacizumab was then explored in the GOG240 phase III trial. It used a $2 \times 2$ factorial design in order to answer two hypothesis: (1) whether the addition of bevacizumab to chemotherapy improves the outcome, and (2) whether the results could be improved with a non-platinum combination based on paclitaxel plus topotecan [8]. The arms were well balanced with respect to stratification factors, including prior concomitant cisplatin and radiation, and stage IVB 'novo'versus recurrent/persistent disease. Cycles were administered every 3 weeks until disease progression, unacceptable toxicity or a complete response was documented (in this case, two additional cycles could be received at their physician's discretion).

This study met its main endpoint by demonstrating an improvement in OS for patients treated with bevacizumab in combination with chemotherapy, compared with chemotherapy alone (17.0 vs 13.3 months; HR 0.71, 95\% CI $0.54-0.95 ; \mathrm{p}=0.0035)$. In addition, the analysis showed that the paclitaxel-topotecan arm was not superior or inferior to the cisplatin-paclitaxel group (median OS $12.5 \mathrm{vs}$ 15 months, respectively; HR 1.2, 95\% CI 0.82-1.76).

The toxicity profile was consistent with the expected side effects associated with bevacizumab. Hypertension of grade 2 or higher was more frequent with bevacizumab-containing regimens $(25 \%$ vs $1.8 \%)$. The rate of grade $\geq 3$ venous thromboembolic events $(8.2 \% \mathrm{vs}$ $1.8 \%$ ), and grade $\geq 2$ gastrointestinal-vaginal and genitourinary fistula $(8.6 \%$ vs $1 \%)$ was higher in the bevacizumab arm.

Other secondary endpoints were also met, and the addition of bevacizumab to chemotherapy led to a clinically meaningful increase in PFS (8.2 months in chemotherapy plus bevacizumab vs 5.9 months in the chemotherapy alone group; HR 0.67, 95\% CI 0.54-0.82; p=0.0002).

Moreover, a statistically significant improvement in overall RR (ORR) was also observed in patients who received bevacizumab in combination with chemotherapy compared with chemotherapy alone (48\% vs 36\%; $\mathrm{p}=0.008)$. Importantly, bevacizumab in combination with chemotherapy did not impact health related quality of life (HRQoL).

\section{Next steps with bevacizumab in cervical cancer}

As previously mentioned, the JGOG 0505 clinical trial established the non-inferiority and better tolerance of the carboplatin-paclitaxel doublet compared with cisplatin-paclitaxel, at least in patients previously treated with cisplatin [5]. In addition, the role of bevacizumab as maintenance after several cycles in combination with chemotherapy has been demonstrated in other tumors; for instance in ovarian cancer (the ICON7 [9], GOG 218 [10] and OCEANS [11] studies).

Based on this rationale, it seemed reasonable to evaluate bevacizumab in combination with the carboplatin/paclitaxel regimen as well as to investigate its role as maintenance therapy after several cycles of chemotherapy. In order to explore both concepts, the CECILIA trial was designed. This study is a multicenter open-label international single arm phase II trial in recurrent, persistent or metastatic cervical cancer. Recruitment was closed after 150 patients from 43 sites across Latin America, Eu- 
rope, the United States and South Africa were enrolled (ClinicalTrials.gov identifier: NCT02467907). The main endpoint was to assess safety, defined by the frequency and severity of gastrointestinal fistula/perforations, gastrointestinal-vaginal fistula and genitourinary fistula in women treated with bevacizumab $15 \mathrm{mg} / \mathrm{kg}$ in combination with paclitaxel $175 \mathrm{mg} / \mathrm{m}^{2}$ and carboplatin AUC 5 every 3 weeks. Chemotherapy for $\geq 6$ cycles was recommended. If toxicity required discontinuation of bevacizumab or chemotherapy, the participant could continue to receive the other ongoing therapy until progression. Secondary endpoints were the evaluation of the overall safety profile of bevacizumab in combination with paclitaxel-carboplatin in this population, the assessment of perforation/fistula events over time and the analysis of

\section{References}

1. Ferlay J, Soerjomataram I, Ervik M et al. GLOBOCAN 2012 v1. 0, Cancer Incidence and Mortality Worldwide: IARC Cancer Base No. 11 2013. Lyon, France: International Agency for Research on Cancer 2014.

2. Moore DH, Blessing JA, McQuellon RP et al. Phase III study of cisplatin with or without paclitaxel in stage IVB, recurrent, or persistent squamous cell carcinoma of the cervix: a Gynecologic Oncology Group study. J Clin Oncol 2004;22(15):3113-9.

3. Long HJ, 3rd, Bundy BN, Grendys EC, Jr. et al. Randomized phase III trial of cisplatin with or without topotecan in carcinoma of the uterine cervix: a Gynecologic Oncology Group Study. J Clin Oncol 2005;23(21):4626-33.

4. Monk BJ, Sill MW, McMeekin DS et al. Phase III trial of four cisplatin-containing doublet combinations in stage IVB, recurrent, or persistent cervical carcinoma: a Gynecologic Oncology Group study. J Clin Oncol 2009;27(28):4649-55.

5. Kitagawa R, Katsumata N, Shibata $T$ et al. Paclitaxel plus carboplatin versus paclitaxel plus cisplatin in metastatic or recurrent cervical cancer: The open-label randomized phase III trial JCOG0505. J Clin Oncol 2015;33(19):2129-35.

6. Monk BJ, Sill MW, Burger RA et al. Phase II trial of bevaci- efficacy (defined by PFS, ORR evaluated by the investigator and OS). The results of this study are expected in the forthcoming months and will add significant evidence for the use of the combination of paclitaxel-carboplatinbevacizumab.

\section{Acknowledgments}

The authors thank Ray Hill, an independent medical writer, who provided native English editing and journal styling on behalf of HPS. This editorial assistance was funded by PharmaMar, Spain.

\section{Conflicts of Interest}

The Authors declare there are no conflicts of interest in relation to this article.

zumab in the treatment of persistent or recurrent squamous cell carcinoma of the cervix: a Gynecologic Oncology Group study. J Clin Oncol 2009;27(7):1069-74.

7. Wright JD, Viviano D, Powell MA et al. Bevacizumab combination therapy in heavily pretreated, recurrent cervical cancer. Gynecol Oncol 2006;103(2):489-93.

8. Tewari KS, Sill MW, Long HJ, 3rd et al. Improved survival with bevacizumab in advanced cervical cancer. N Engl J Med 2014;370(8):734-43.

9. Oza AM, Cook AD, Pfisterer J et al. Standard chemotherapy with or without bevacizumab for women with newly diagnosed ovarian cancer (ICON7): overall survival results of a phase 3 randomised trial. Lancet Oncol 2015;16(8):928-36.

10. Burger RA, Brady MF, Bookman MA et al. Incorporation of bevacizumab in the primary treatment of ovarian cancer. N Engl J Med 2011;365(26):2473-83.

11. Aghajanian C, Blank SV, Goff BA et al. OCEANS: a randomized, double-blind, placebo-controlled phase III trial of chemotherapy with or without bevacizumab in patients with platinum-sensitive recurrent epithelial ovarian, primary peritoneal, or fallopian tube cancer. J Clin Oncol 2012;30(17):2039-45. 